\title{
Update on the Management of Diabetic Macular Edema
}

\author{
Shawn M Iverson' and W Lloyd Clark',2 \\ 1. Palmetto Health - University of South Carolina Medical Group, Columbia, South Carolina, US; 2. Assistant Clinical Professor of Ophthalmology, \\ University of South Carolina School of Medicine, Palmetto Retina Center LLC, West Columbia, South Carolina, US
}

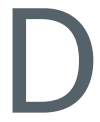
iabetic macular edema (DME) is a treatable sequela of diabetic retinopathy and a significant cause of visual morbidity among working age individuals worldwide. While anti-vascular endothelial growth factor (anti-VEGF) agents are first-line agents in the management of DME, corticosteroids and laser therapy can play a role as well. Despite a growing understanding of best clinical practices, many patients respond unpredictably to therapy. This article will briefly review current treatment modalities and discuss future treatment options for managing DME.

\section{Keywords}

Diabetic macular edema, anti-vascular endothelial growth factor, VEGF, macular laser, aflibercept, bevacizumab, ranibizumab

Disclosure: Shawn M Iverson has nothing to disclose in relation to this article. W Lloyd Clark has received consulting fees from Bayer, Genentech/Roche, Regeneron; speakers bureau from Genentech/Roche, Regeneron; contracted research: Allergan, Inc., Genentech/Roche, Regeneron; and equity from VERSYL, Inc. No funding was received in the publicaiton of this article. This study involves a review of the literature and did not involve any studies with human or animal subjects performed by any of the authors.

Authorship: All named authors meet the International Committee of Medical Journal Editors (ICMJE) criteria for authorship of this manuscript, take responsibility for the integrity of the work as a whole, and have given final approval to the version to be published.

open Access: This article is published under the Creative Commons Attribution Noncommercial License, which permits any noncommercial use, distribution, adaptation, and reproduction provided the original author(s) and source are given appropriate credit.

Received: January 10, 2017

Accepted: February 15, 2017

Citation: US Ophthalmic Review, 2017;10(1):52-6

Corresponding Author: Lloyd Clark, MD, Palmetto Retina Center, 124 Sunset Court, West Columbia, SC 29169 US. F: LClark@PalmettoRetina.com
Diabetic retinopathy is the leading cause of blindness worldwide among patients aged 20-74. ${ }^{\text {Among }}$ eyes with diabetic retinopathy, diabetic macular edema (DME) accounts for a significant proportion of vision loss and associated morbidity, ${ }^{2,3}$ and the reported incidence is quite high, occuring in $26.1 \%$ of type 1 diabetics according to The Wisconsin Epidemiologic Study of Diabetic Retinopathy. ${ }^{4}$ Tight glucose control significantly reduces the risk of development and progression of diabetic retinopathy in both type 1 and type 2 diabetics, and this remains the best preventative measure. ${ }^{5-7}$ These studies have additionally shown other modifiable risk factors, such as hypertension control, play important roles in managing diabetic eye disease. For those eyes that do develop macular edema, a number of treatment options exist, and research into new and modified modalities is ongoing. Recently, firstline therapy for DME has shifted and patients and clinicians can expect visual improvement with consistent treatment. As research advances, it is likely that new therapies will emerge and preferred practice patterns will continue to evolve.

\section{Anti-vascular endothelial growth factor Background}

Anti-vascular Endothelial Growth Factor (anti-VEGF) agents have become stardard of care in the treatment of center-involving DME. The first anti-VEGF agent used in treating DME was pegaptanib (Macugen ${ }^{\circledR}$, Pfizer, New York, US), a pegylated anti-VEGF aptamer. In the early 2000s, a phase ॥ study was completed showing pegaptanib reduces macular thickness and improves vision, ${ }^{8}$ however, the development of this agent did not move forward as more efficacious anti-VEGF agents came to market. Today, clinicians choose from three commercially available anti-VEGF agents for treating DME and for treating diabetic retinopathy associated with DME; bevacizumab (Avastin ${ }^{\circledR}$, Genentech, California, US), ranibizumab (Lucentis ${ }^{\oplus}$, Genentech, California, US), and aflibercept (Eylea ${ }^{\circledR}$, Regeneron, New York, US), the latter two of which have Food and Drug Administration (FDA) approval.

Bevacizumab, ranibizumab, and alfibercept are all anti-VEGF medications, however there are notable structural differences between these agents. Bevacizumab is a recombinant, humanized, full length monoclonal antibody to VEGF. Ranibizumab is a monoclonal antibody antigen-binding fragment to VEGF. Ranibizumab is unique in that it lacks an Fc portion, which results in a more rapid clearance from the body, ${ }^{9}$ a characteristic some believe plays a role in differences in systemic adverse effects, although this is not conclusively proven. Aflibercept is a recombinant fusion protein consisting of binding domains to VEGF-receptors 1 and 2 bound to the FC portion human immunoglobulin G1 (IgG1). These structural differences affect binding affinity for VEGF, which plays a role in differences in in vivo activity and clinical response. Analyzing binding affinity is highly complex and has been studied with mixed results. ${ }^{10,11}$ While aflibercept is thought to be more potent, a recent study by 
Yang et al., found that both alfibercept and ranibizumab, under uniform conditions, are equipotent in their ability to inhibit VEGF, and both had significantly greater binding affinity compared to bevacizumab.? Nevertheless, all three agents significantly reduce intraocular VEGF levels.

\section{Efficacy}

There is extensive literature reporting clinical outcomes using bevacizumab, ranibizumab and aflibercept; however, most of the controlled clinical trials have been performed using ranibizumab or aflibercept. ${ }^{12-18}$ Although a detailed review of the many anti-VEGF studies is beyond the scope of this article, the Diabetic Retinopathy Clinical Research Network (DRCR.net) Protocol T is worth mentioning as a sentinel study that prospectively compared all three agents for treating DME in a randomized clinical trial. The primary endpoint for Protocol T was mean change in visual acuity at 1-year, and this study reported all medications improved vision and decreased macular thickness. However, at worse levels of baseline vision (i.e., $\leq 20 / 50$ ), the aflibercept group obtained a statistically significant improvement in visual acuity letter score compared to the other agents. ${ }^{18}$ Two-year outcomes have since been published showing the visual acuity improvement (12.8, 10.0, and 12.3 letters) and number of injections $(15,16,15)$ over 2 years was similar between the aflibercept, bevacizumab and ranibizumab groups, repectively. Visual acuity improvements were greater among eyes with baseline visual acuity $\leq 20 / 50,18.1,13.3$, and 16.1 letters, respectively, with aflibercept remaining superior to bevacizumab $(p=0.02)$, however the statistical superiority over ranibizumab that was observed in 1 year data was no longer observed $(\mathrm{p}=0.18) .{ }^{15}$ Protocol $\mathrm{T}$ also showed a significant reduction in central subfield thickness (CST) among all three agents at 2-years, with the greatest reduction in the aflibercept group $(-211 \pm 155)$, followed by ranibizumab $(-185 \pm 158)$ and bevacizumab $(-174 \pm 159)$ groups. While Protocol T was a well designed, large, randomized, prospective study, all studies have limitations. The main limitations of Protocol $T$ include the drugs not being used according to their labeled dosing strategy and that this is a single trial rather than parallel trials required for FDA registration. An additional study of similar nature would be of great benefit to confirm Protocol T's findings, but to date no study has been organized. There are additional anti-VEGF studies evaluating these agents in various scenarios, and all three medications continue to represent reasonable choices.

\section{Safety}

Anti-VEGF agents have a remarkable safety profile and have been administered in tens of thousands of eyes with DME and other conditions such as age-related macular degeneration, retinal vein occlusions, and to a lesser degree in conditions such as juxtafoveal macular telangiectasia, central serous chorioretinopathy, and retinopathy of prematurity. Serious adverse events are relatively uncommon and include: sterile intraocular inflammation, rhegmatogenous retinal detachment, sustained intraocular pressure (IOP) elevation, ocular hemorrhage, endophthalmitis, and various systemic events. All anti-VEGF agents have been associated with detectable levels in the systemic circulation; however, systemic event rates vary by trial and by anti-VEGF agent. The DRCR.net Protocol T reported the rates of Anti-platelet Trialists' Collaboration (APTC) events, and found they occurred in $5 \%$ with aflibercept, $8 \%$ with bevacizumab and $12 \%$ with ranibizumab, with a statistically significant increased rate in ranibizumab versus aflibercept ( $p=0.047)$, but not between the other groups. It is unclear if this increased APTC rate in ranibizumab eyes was a chance finding since it has not been observed in other protocols.
There are other adverse events that have been reported specifically in patients with diabetic retinopathy, all of which are quite rare. Multiple groups have reported progression of tractional retinal detachment in advanced proliferative diabetic retinopathy (PDR) eyes, ${ }^{19-21}$ and ghost cell glaucoma when bevacizumab was used as an adjunct to vitrectomy in PDR. ${ }^{22}$ Many clinicians continue to administer an anti-VEGF agent prior to vitrectomy in PDR, however there is no consensus on the optimal timing between these two procedures.

Despite ample research and large meta-analyses demonstrating little systemic risk with anti-VEGF treatments in most patients, ${ }^{23-27}$ clinicians should be mindful of these agents in this higher-risk population. A recent meta-analysis looking specifically at diabetic patients with intensive (monthly for 2 years) intravitreal anti-VEGF injections found an increased risk of death and potentially cerebrovascular accidents, although not myocardial infarction or arteriothrombotic events, in this high-risk group. ${ }^{28}$ It remains up to the discretion of the clinician when to continue or hold doses of anti-VEGF, as no specific guidelines exist.

\section{Selecting an anti-VEGF agent}

What we have learned from Protocol $\mathrm{T}$ is, from a clinical standpoint, there is no single agent that is vastly superior to others, and all three agents represent reasonable options for treating DME. Cost, baseline visual acuity, medication availability, shelf life, and yet to be determined factors will continue to play roles in selecting an agent. The American Society of Retinal Specialist convened a group of experts to provide their perspective on the clinical findings of Protocol $T$ and stated that all three agents result in improved vision, and "many specialists might initiate therapy with bevacizumab when visual acuity is good (i.e., 20/32 to 20/40 as measured in the DRCR Network), recognizing that the cost-effectiveness of bevacizumab outweighs that of aflibercept or ranibizumab". ${ }^{29,30}$ Data from Protocol $T$ do suggest aflibercept is a more efficacious anti-VEGF medication among eyes with worse baseline visual acuity (i.e., $\leq 20 / 50$ ). Additionally, it should be noted that the value of more rapid early visual acuity and anatomic improvements on long-term outcomes is not yet completely understand, and it is possible that 5- and 10-year studies will show greater discordance in visual acuity outcomes between anti-VEGF medications. Furthermore, certain patient factors, such as inability to return to clinic regularly or systemic comorbidities such as recent vasuclar events, may be relative contraindications to anti-VEGF therapy and altogether persuade clinicians into using a non-anti-VEGF treatment modality.

\section{Corticosteroid agents Background}

VEGF certainly plays a major role in the etiology of DME; however, other factors are at play. The pathogenesis of DME includes breakdown of the blood-retinal barrier and an increase in the concentration of numerous cytokines and other inflammatory mediators. ${ }^{31}$ Corticosteroids do affect VEGF levels, but additionally are potent modulators of these other inflammatory mediators, and as such, can be used to treat DME. Corticosteroids initially gained popularity when outcomes with laser were suboptimal and the first report of intraocular crystalline corticosteroid injection for the treatment of DME was published in 2001. ${ }^{32}$ There are now three fluorinated synthetic corticosteroids that lack mineralocorticoid activity used in clinical practice. Dexamethasone as a sustained release biodegradable implant (DEX implant; Ozurdex ${ }^{\circledR}$, Allergan Inc., California, 
US) and fluocinolone as a sustained release nonbiodegradable implant (Iluvien ${ }^{\circledR}$, Alimera Sciences, Georgia, US) are FDA approved for treating DME. Triamcinolone in an ophthalmic formulation is used off-lable as an intravitreal or subTenon's injection for DME. Steroid use initially fell out of favor with the introduction of anti-VEGF agents, however, more recently, use has been increasing when there is an inadequate or incomplete response to anti-VEGF monotherapy.

\section{Efficacy}

Over the years, several studies have been conducted to investigate the utility of corticosteroids for the treatment of DME. The DRCR.net Protocol B found at 2 years, treatment with focal/grid photocoagulation was more effective and had fewer adverse effects than $1 \mathrm{mg}$ or $4 \mathrm{mg}$ doses of intravitreal triamcinolone acetonide. ${ }^{33}$ The DRCR.net Protocol E randomized patients to laser and or subTenon's triamcinolone and found no additional benefit to subTenon's steroid. ${ }^{34}$ In a randomized, double-masked, placebo-controlled trial, Gillies et al. reported, at 2 years, intravitreal triamcinolone improved vision and reduced macular thickness in eyes with DME refractory to macular laser. ${ }^{35}$ Following this, DRCR.net Protocol I evaluated both intravitreal triamcinolone acetonide and ranibizumab in combination with early and deferred macular photocoagulation, and found that at 2 years, the steroid group outcomes were substantially inferior to the intravitreal ranibizumab with or without photocoagulation or photocoagulation alone groups. ${ }^{36}$ Subanalysis of Protocol I data limited to eyes that were pseudophakic at baseline did show similar improvements in visual acuity compared to the ranibizumab arm, however the steroid arm had an increased rate of IOP elevation. Ozurdex typically has a duration of effect between 3-6 months, and the MEAD Study Group has shown with 4-5 injections over a 3-year period, a significant proportion of patients had greater than 100 micron reduction in macular thickness and an associated 5.9 and 6.5 letter gain in the 0.35 mg and 0.7 mg dosing groups, respectively..$^{37}$ Similarly, the Iluvian ${ }^{\circledR}$ implant (Alimera Sciences, Inc., Georgia, US) has been shown to be effective; however, this nonbiodegradable implant has been shown to elute drug for up to 3 years. ${ }^{38}$ Both implants result in cataract formation in a significant number of phakic patients and this finding confounds visual acuity outcomes. When adjusting for cataract formation, intravitreal steroids (via simple injection or sustained release implant) have been shown to have similar visual acuity efficacy as anti-VEGF agents. ${ }^{36,39}$

\section{Safety}

It is well known that corticosteroids accelerate cataract formation. DRCR. netProtocol $\mathrm{B}$ found the cumulative probability of cataract surgery at 3 years was $46 \%$ and $83 \%$ in the 1 and 4 mg steroid groups, both notably higher than the $31 \%$ in the laser group. ${ }^{33}$ The Ozurdex MEAD Study Group reported cataracts in $67.9 \%, 64.1 \%$, and $20.4 \%$ of the $0.7 \mathrm{mg}, 0.35 \mathrm{mg}$ and sham eyes at 3 years, respectively. ${ }^{37}$ The FAME Study Group found almost all phakic eyes developed cataracts by 3 years follow-up, with rates of $81.7 \%, 88.7 \%, 50.7 \%$ in the low-dose, high-dose and sham groups, respectfully. ${ }^{38}$ There is also a significant risk of IOP response with steroid use and a recent meta-analysis looking at ocular hypertension following intravitreal administration found $11-79 \%$ of eyes develop pressure elevation depending on the agent and dose used..$^{40}$ One study found roughly one-third of eyes receiving the dexamethasone implant $27.7 \%$ in the high-dose and $24.8 \%$ in the low-dose) had an IOP increase $\geq 10 \mathrm{mmHg}$ from baseline. ${ }^{37}$ Similarly, the fluocinolone implant is associated with high rates of IOP elevations, with rates of $37.1 \%, 45.5 \%$, and $11.9 \%$ in the low-dose, high-dose and sham groups, respectively. ${ }^{38}$ Despite this significant incidence of IOP response, most eyes can be controlled medically. Among studies specifically for diabetic macular edmea and following patients for at least 2 years, incisional glaucoma surgery rates have been reported to be between $0-8 \% .{ }^{36-39}$

\section{Selecting an agent}

There are a number of corticosteroid agents and administration methods for treating DME and all have advantages and disadvantages. Topical corticosteroids are not typically used to treat DME as they do not readily reach therapeutic concentrations in the posterior segment; however, this route may allow for identification of IOP responders. SubTenon's injections results in reasonable posterior segment concentrations and allow for identification of IOP response, but evidence suggests this route is not as effective as intravitreal injection. Intravitreal injections are done with triamcinolone acetonide or via implantation of a sustained release device.

Today, our recommended approach for patients with suboptimal antiVEGF response or those with unacceptable treatment burden would be to use triamcinolone (Triesence ${ }^{\circledR}$, Alcon, Texas, US) or Ozurdex to look for treatment and steroid response, and if favorable, consider iluvien. We additionally advise clinicians counsel phakic patients on the high likelihood developing a visually significant cataract which may require surgery.

\section{Macular laser treatment Background}

Macular grid and focal laser photocoagulation have been used for decades and this became the standard treatment for clinically significant DME after results of the Early Treatment Diabetic Retinopathy Study (ETDRS) were published in the late 1980 's. ${ }^{41}$ A number of laser platforms are available, all of which ultimately result in the laser-tissue interaction termed photocoagulation. This results in closure of leaking microaneurysms and in theory decrease oxygen demand through destruction of oxygenconsuming photoreceptors. Laser systems have evolved and now include lasers of different wavelengths, micropulsed lasers and navigable lasers, each of which has certain advantages. Many laser systems generate a single wavelength, however systems are available that allow selection from multiple color options. Some clinicians prefer yellow lasers because yellow light seems to be more selective for retinovascular lesions and may produce less scar formation; however, no large studies have proven differences between wavelengths affects clinical outcomes. Micropulsed lasers reduce heat transfer compared to a continuous wave, resulting in less tissue destruction and treatment without a visibly identifiable burn. Navigable lasers employ eye tracking and angiography-guidance software that improve accuracy and speed of treatment; however, cost continues to limited adoption of this technology.

\section{Efficacy}

A number of clinical trails have evaluated the outcomes of focal and or grid photocoagulation for the treatment of DME. The ETDRS was a sentinel study that evaluated both focal and early scatter photocoagulation for treating DME. ${ }^{41}$ This study found focal macular laser halved the rate of moderate vision loss (three or more lines) and increased the chance of moderate vision gain and reduced retinal thickening compared to a control group of untreated eyes. The ETDRS and other studies showed macular laser is effective in reducing vision loss; however, the introduction of anti- 
VEGF resulted in a paradigm shift in treatment. Studies such as DRCR.net Protocol I, 36,42 VISTA (Study of Intravitreal Administration of VEGF Trap-Eye [BAY86-5321] in Patients with Diabetic Macular Edema) ${ }^{43}$ and VIVID (VEGF Trap-Eye in Vision Impairment Due to DME), ${ }^{43}$ have demonstrated clear superiority of anti-VEGF over macular laser, and as such, anti-VEGF has become the first line treatment standard.

Macular laser is still utilized in clinical practice and is being investigated for its role as an adjunct treatment. The TREX-DME study (Treat and Extend Protocol using Ranibizumab with and without Laser Photocoagulation for Diabetic Macular Edema) compared monthly dosing of treat and extend ranibizumab $0.3 \mathrm{mg}$ with and without angiography-guided macular laser photocoagulation, to determine the effect of laser on antiVEGF injection number. At 1 year, TREX-DME did not find a significant difference between these two groups, reporting a similar number of yearly injections between the treat and extend ranibizumab (mean 10.7 injections) and treat and extend ranibizumab with guided laser (mean 10.1 injection) groups. ${ }^{44}$ Despite the findings of this study, we know from clinical experience and numerous prior studies that macular laser does decrease edema, and more research is required to determine how focal and grid macular laser impact visual outcomes in the setting of anti-VEGF treatment.

\section{Safety}

Macular laser is a very safe procedure when performed correctly; however, it is not without risks. Devastating vision loss can occur if laser is applied to the fovea. The risk of this complication can be mitigated by appropriate identification of landmarks and good patient instruction. Recent advances in technology have lead to eye tracking and angiography-guided laser treatment. The Navilas ${ }^{\circledR}$ laser platform (OD-OS $\mathrm{GmbH}$, Teltow, Germany) is a navigable laser whereby imaging technology identifies pathology such as microaneurysms. The physician can choose which lesions to treat, and a laser eye-tracking and angiographic-guiding software allow precise and rapid targeting of pathology, which may improve safety and efficacy. ${ }^{45}$

Other risks include rupture of Bruch's membrane, which occurs when high fluence is used (i.e., high power over a small area), which can lead to hemorrhage and choroidal neovascularization. Additionally, laser spots may produce small scotomas, although most often these are imperceptible to the patient. Notable advantages of laser over intravitreal injection of either anti-VEGF or corticosteroids is the elimination of risks associated with the injection procedure and medication related adverse effects.

\section{When to use macular laser}

Laser therapy remains widely available and a beneficial option in certain scenarios. Good candidates include those with DME that do not qualify for anti-VEGF, such as patient with clinically significant macular edema but no central thickening or visual acuity loss. Macular laser should also be considered in patients with leaking microaneurysms outside the fovea but with suboptimal response or contraindication to anti-VEGF. Laser therapy may also be the preferred choice when follow-up compliance is a concern or to reduce vision loss in those that cannot return for additional treatments. Future studies will help further characterize the conditions under which macular laser offers the greatest benefit as an adjunctive treatment.

\section{Combination treatments and future treatment options}

The current consensus first-line treatment for vision loss due to DME is intravitreal injection of an anti-VEGF agent. Treatment with these drugs results in a rapid reduction in macular thickness and often results in significant visual acuity improvement. Despite anti-VEGF's favorable safety and efficacy profile, there are still several downsides, notably high treatment cost and visit burden. While several studies have found a decreased dependence on injections over time, patients still require fairly frequent follow-up, as reaccumulation of retinal fluid is not uncommon when injections are stopped. A number of ongoing studies are looking at new agents and combination therapies to evaluate the optimal regimen for visual and anatomical outcomes while limiting the frequency of treatments.

The DRCR network continues to actively investigate DME treatments through many study protocols. Protocol U (ClinicalTrials.gov Identifier: NCT01945866) is currently in phase II and was designed to evaluate visual acuity and retinal thickness outcomes in eyes receiving an intravitreal dexamethasone implant plus anti-VEGF versus sham plus anti-VEGF. corticosteroid and anti-VEGF combination may have a synergistic effect by dramatically reducing levels of VEGF, cytokines and other inflammatory mediators. Protocol V (ClinicalTrials.gov Identifier: NCT01909791) is currently recruiting participants to investigate prompt anti-VEGF versus prompt laser plus deferred anti-VEGF versus observation plus deferred anti-VEGF among eyes with good vision ( $\geq 20 / 25$ ), with no or minimal prior treatment for DME. Protocol W (ClinicalTrials.gov Identifier: NCT02634333) is looking at patients without center involving DME but with severe nonPDR (NPDR) to determine the safety and efficacy of prompt anti-VEGF versus observation for prevention of vision-threatening outcomes. Similarly, Panorama (ClinicalTrials.gov Identifier NCT02718326) is a study, currently in phase III, comparing anti-VEGF (aflibercept) versus sham in patients with moderately severe to severe NPDR to determine the effect on retinopathy, vision and the development of DME.

Newer anti-VEGF agents are being developed that display higher potency for binding VEGF.10 Given the relatively high affinity of some currently available agents, it remains to be seen whether targeting these additional proteins will offer any improved or prolonged efficacy over currently available agents. In addition to new anti-VEGF molecules, other molecules that can retard vascular growth and leakage are also being investigated. Anti-angiopoietin is currently being evaluated for its ability to increase the anti-leak effect of anti-VEGF agents (ClinicalTrials.gov Identifier: NCT02712008). Aerpio AKB-9778 is a Tie-2 activating agent that effectively blocks vascular leak and pathologic angiogenesis in multiple disease conditions, and this agent is also being investigated for the treatment of DME (ClinicalTrials.gov Identifier: NCT02050828).

Besides new anti-angiogenic/anti-leak agents being developed, new delivery systems could potentially be developed. Slow-release anti-VEGF implants are being investigated for use in neovascular age-related macular degeneration, however to our knowledge, no devices are currently in any phase study for DME in the US.

As mentioned above, macular laser is still being investigated to determine the role of laser in DME. TREX-DME has reported 1-year data, ${ }^{44}$ and 2-year data will be available soon. Similarly, investigators are looking at the role of 
micropulsed laser on the treatment burden of anti-VEGF in the Micropulsed Laser in Association With Anti-VEGF for the Treatment of Macular Edema in Diabetic Patients (LAMED) study (ClinicalTrials.gov Identifier: NCT02650050).

Many advances in treating DME have been made over the past decades and it is imperative that this area of research remains well funded, as diabetic eye disease is the leading cause of blindness in the working age individual. Anti-VEGF therapy remains first-line; however, it is necessary for any physician treating DME to have other options in their armamentarium. There are several clinical trials investigating a number of treatment options, and it is likely that a combination of treatments will provide the best balance of safety, efficacy, cost, and treatment burden. $\square$
1. Resnikoff $S$, Pascolini $D$, Etya'ale $D$, et al., Global data on visual impairment in the year 2002. Bull World Health Organ, 2004:82:844-51.

2. Hariprasad SM, Mieler WF, Grassi M, et al., Vision-related quality of life in patients with diabetic macular oedema, $\mathrm{Br} J$ Ophthalmol, 2008;92:89-92

3. Lamoureux EL, Tai ES, Thumboo J, et al., Impact of diabetic retinopathy on vision-specific function, Ophthalmology, 2010;117:757-65

4. Klein R, Knudtson MD, Lee KE, et al., The Wisconsin Epidemiologic Study of Diabetic Retinopathy XXIII: the twenty-five-year incidence of macular edema in persons with type 1 diabetes, Ophthalmology, 2009;116:497-503.

5. Diabetes Control and Complications Trial Research Group, The effect of intensive treatment of diabetes on the development and progression of long-term complications in insulin-dependent diabetes mellitus, N Eng/ I Med, 1993:329:977-86.

6. Diabetes Control and Complications Trial Research Group, Progression of retinopathy with intensive versus conventiona treatment in the Diabetes Control and Complications Tiral, Ophthalmology, 1995;102:647-61.

7. UK Prospective Diabetes Study Group, Intensive blood glucose control with sulphonylureas or insulin compared with conventional treatment and risk of complications in patients with type 2 diabetes (UKPDS 33) Lancet, 1998:352:837-53.

8. Cunningham ET Jr, Adamis AP, Altaweel M, et al., Macugen Diabetic Retinopathy Study Group, A phase II randomized doublemasked trial of pegaptanib, an anti-vascular endothelial growth factor aptamer, for diabetic macular edema, Ophthalmology, 2005;112:1747-57.

9. Avery RL, Castellarin AA, Steinle NC, et al., Systemic pharmacokinetics following intravitreal injections of ranibizumab, bevacizumab, or aflibercept in patients with neovascular AMD, $B$ .

10. Yang J, Wang $X$, Fuh $G$, et al., Comparison of binding characteristics and in vitro activities of three inhibitors of vascular endothelial growth factor, A Mol Pharm, 2014 6;11:3421-30.

11. Papadopoulos N, Martin J, Ruan Q, et al., Binding and neutralization of vascular endothelial growth factor (VEGF) and related ligands by VEGF Trap, ranibizumab and bevacizumab, Angiogenesis, 2012;15:171-85

12. Nguyen $\mathrm{QD}$, Shah SM, Heier JS, et al., Primary end point (six months) results of the Ranibizumab for Edema of the macula in diabetes (READ-2) study, Ophthalmology, 2009;116:2175-81.

13. Korobelnik JF, Do DV, Schmidt-Erfurth U, et al., Intravitrea aflibercept for diabetic macular edema, Ophthalmology 2014;121:2247-54.

14. Scott IU, Edwards AR, et al., Diabetic Retinopathy Clinica Research Network, A phase II randomized clinical tria of intravitreal bevacizumab for diabetic macular edema Ophthalmology, 2007:114:1860-7.

15. Wells JA, Glassman AR, Ayala AR, et al., Diabetic Retinopathy Clinical Research Network, Aflibercept, bevacizumab, or ranibizumab for diabetic macular edema: two-year results from a comparative effectiveness randomized clinical trial, Ophthalmology, 2016;123:1351-9.

16. Nguyen QD, Brown DM, Marcus DM, et al., RISE and RIDE Research Group, Ranibizumab for diabetic macular edema: results from 2 phase III randomized trials: RISE and RIDE, Ophthalmology ، 2012:119:789-801.

17. Brown DM, Schmidt-Erfurth U, Do DV, et al., Intravitreal aflibercept for diabetic macular edema: 100-week results from the VISTA and VIVID studies, Ophthalmology, 2015;122:2044-52.

18. Wells JA, Glassman AR, Ayala AR, et al., Diabetic Retinopathy Clinical Research Network, Aflibercept, bevacizumab, or ranibizumab for diabetic macular edema, N Eng/ I Med, 2015:372:1193-203.

19. Arevalo JF, Maia M, Flynn Jr HW, et al., Tractional retina detachment following intravitreal bevacizumab (Avastin) in patients with severe proliferative diabetic retinopathy, $\mathrm{Br} J$ Ophthalmol, 2008;92:213-6.

20. Oshima Y, Shima C, Wakabayashi T, et al., Microincision vitrectomy surgery and intravitreal bevacizumab as a surgical adjunct to treat diabetic traction retinal detachment, Ophthalmology, 2009;116:927-38

21. Yeh PT, Yang CM, Lin YC, et al., Bevacizumab pretreatment in vitrectomy with silicone oil for severe diabetic retinopathy, Retina, 2009;29:768-74.

22. Liu L, Wu WC, Yeung L, et al., Ghost cell glaucoma after intravitrea bevacizumab for postoperative vitreous hemorrhage following vitrectomy for proliferative diabetic retinopathy, Ophthalmic Surs Lasers Imaging, 2010;41:72-7.

23. Avery RL, Francom S, Lai P, et al., Meta-analysis examining the systemic safety profile of intravitreal ranibizumab injections in Systemic safety profile of intravitreal ranibizumab injections in

24. Ford JA, Lois N, Royle P, et al., Current treatments in diabetic macular oedema: systematic review and meta-analysis, BMJ Open, 2013;3:e002269.

25. ThulliezM, Angoulvant $\mathrm{D}$, Le LezML, et al., Cardiovascular events and bleeding risk associated with intravitrea antivascular endothelial growth factor monoclonal antibodies: systematic review and meta-analysis, JAMA Ophthalmol, 2014:132:1317-26.

26. Ueta T, Noda Y, Toyama T, et al., Systemic vascular safety of ranibizumab for age-related macular degeneration: systematic review and meta-analysis of randomized trials, Ophthalmology 2014; 121:2193-203

27. Yanagida Y, Ueta T, Systemic safety of ranibizumab for diabetic macular edema: meta-analysis of randomized trials, Retina 2014;34:629-35

28. Avery RL, Gordon GM, Systemic Safety of Prolonged Monthly Anti-Vascular Endothelial Growth Factor Therapy for Diabetic Macular Edema: A Systematic Review and Meta-analysis, JAMA Ophthalmol, 2016;134:21-9.

29. Heier JS, Bressler NM, Avery RL, et al., American Society of Retina Specialists Anti-VEGF for Diabetic Macular Edema Comparative Effectiveness Panel. Comparison of aflibercept, bevacizumab and ranibizumab for treatment of diabetic macular edema: extrapolation of data to clinical practice, JAMA Ophthalmol, 2016:134:95-9.

30. Ross EL, Hutton DW, Stein JD, et al., Diabetic Retinopathy Clinical Research Network, Cost-effectiveness of aflibercep bevacizumab, and ranibizumab for diabetic macular edema treatment: analysis from the diabetic retinopathy clinical research network comparative effectiveness trial, JAMA Ophthalmol 2016;134:888-96.
31. Sohn HJ, Han DH, Kim IT, et al., Changes in aqueous concentrations of various cytokines after intravitreal triamcinolone versus bevacizumab for diabetic macular edema Am I Ophthalmol, 2011;152:686-94.

32. Jonas JB, Sofker A, Intraocular injection of crystalline cortisone as adjunctive treatment of diabetic macular edema, $\mathrm{Am}$ 」 Ophthalmol, 2001;132:425-7.

33. Beck RW, Edwards AR, Aiello LP, et al., Diabetic Retinopathy Clinical Research Network (DRCR.net), Three-year follow-up of a randomized trial comparing focal/grid photocoagulation and intravitreal triamcinolone for diabetic macular edema, Arch Ophthalmol, 2009;127:245-51.

34. Chew E, Strauber $S$, Beck R, et al., Diabetic Retinopathy Clinical Research Network, Randomized trial of peribulbar triamcinolone acetonide with and without focal photocoagulation for mild diabetic macular edema: a pilot study, Ophthalmology, 2007:114:1190-6.

35. Gillies MC, Sutter FK, Simpson JM, et al., Intravitreal triamcinolone for refractory diabetic macular edema: two-year results of a double-masked, placebo-controlled, randomized clinical trial Ophthalmology, 2006;113:1533-8.

36. Elman MJ, Bressler NM, Qin H, et al., Diabetic Retinopathy Clinical Research Network, Expanded 2-year follow-up of ranibizumab plus prompt or deferred laser or triamcinolone plus prompt laser for diabetic macular edema, Ophthalmology, 2011:118:609-14.

37. Boyer DS, Yoon YH, Belfort R Jr, et al., Ozurdex MEAD Study Group, Three-year, randomized, sham-controlled trial of dexamethasone intravitreal implant in patients with diabetic macular edema, Ophthalmology, 2014;121:1904-14

38. Campochiaro PA, Brown DM, Pearson A, et al., FAME Study Group, Sustained delivery fluocinolone acetonide vitreous inserts provide benefit for at least 3 years in patients with diabetic macular edema, Ophthalmology, 2012 Oct;119:2125-32.

39. Fraser-Bell S, Lim LL, Campain A, et al., Bevacizumab or Dexamethasone Implants for DME: 2-year Results (The BEVORDEX Study), Ophthalmology, 2016;123:1399-401.

40. Kiddee W, Trope GE, Sheng L, et al., Intraocular pressure monitoring post intravitreal steroids: a systematic review, Surv Ophthalmol, 2013;58:291-310.

41. Early Treatment Diabetic Retinopathy Study Research Group, Treatment techniques and clinical guidelines for photocoagulation of diabetic macular edema. ETDRS Report number 2 Ophthalmology, 1987:94:761-74.

42. Elman MJ, Aiello LP, Beck RW, et al., Diabetic Retinopathy Clinica Research Network (DRCR.net), Randomized trial evaluating ranibizumab plus prompt or deferred laser or triamcinolone plus prompt laser for diabetic macular edema, Ophthalmology, 2010;117:1064-77.

43. Korobelnik JF, Do DV, Schmidt-Erfurth U, et al., Intravitreal aflibercept for diabetic macular edema, Ophthalmology 2014:121:2247-54.

44. Payne JF, Wykoff CC, Clark WL, et al., TREX-DME Study Group, Randomized Trial of Treat and Extend Ranibizumab with and without Navigated Laser for Diabetic Macular Edema: TREX-DME 1 Year Outcomes, Ophthalmology, 2017;124:74-8.

45. Kozak I, Oster SF, Cortes MA, et al., Clinical evaluation and treatment accuracy in diabetic macular edema using navigated laser photocoagulator NAVILAS, Ophthalmology, 2011;118:1119-24. 